\title{
Regular and Equiangular Polygons of a Hyperbolic Plane of Positive Curvature
}

\author{
Lyudmila N. Romakina \\ (Communicated by Murat Tosun)
}

\begin{abstract}
A hyperbolic plane $\widehat{H}$ of positive curvature can be realized on the ideal domain of a Lobachevskii plane. The plane $\widehat{H}$ is the projective Cayley-Klein model of a two-dimensional de Sitter space. It is shown that on $\widehat{H}$ there are no finite regular polygons topologically equivalent to a disk. A finite equiangular polygon homeomorphic to a disk is a quadrangle with hyperbolic quasiangles. In the plane $\widehat{H}$ we study also equiangular and regular polygons homeomorphic to the Möbius band. It is proved that the interior angle at the vertex of a finite equiangular Möbius polygon of the plane $\widehat{H}$ is a non-convex elliptic pseudoangle.
\end{abstract}

Keywords: hyperbolic plane of positive curvature; generalized polygon; generalized Möbius polygon; regular polygon; equiangular polygon.

AMS Subject Classification (2010): Primary: 51M10; Secondary: 51M09; 51M16; 51M20; 51M25; 51F05.

This paper is devoted to the 225-th anniversary of the birth of Nikolai Ivanovich Lobachevsky

\section{Introduction}

Let $H^{2}$ be an extended hyperbolic plane with absolute oval curve $\gamma$ (see [22, §5.1.1]). The plane $H^{2}$ has three types of lines. Lines intersecting the absolute in two real (imaginary conjugate) points are called hyperbolic (elliptic). Any isotropic line on $\mathrm{H}^{2}$ tangent to the absolute is called parabolic.

In the Cayley - Klein model, the interior domain with respect to the oval curve $\gamma$ is a complete Lobachevskii plane $\Lambda^{2}$, that is, a hyperbolic plane of negative curvature. On the exterior domain with respect to the absolute curve $\gamma$ one can construct different geometries. If we consider hyperbolic, elliptic, and parabolic lines as lines in our geometry, we obtain a hyperbolic plane $\widehat{H}$ of positive curvature (see [8], [9], [23, §4.1.1]). Geometry of the plane $\widehat{H}$ of curvature $1 / \rho^{2}$ can be realized in the pseudo-Euclidean space $\mathbb{R}_{1}^{3}$ on the sphere of real radius $\rho$ with antipodal points identified. Therefore the plane $\widehat{H}$ is the projective model of two-dimensional de Sitter space (see, for instance, [4], [24]). The number $\rho$ is called the curvature radius of $\widehat{H}$. The plane $\widehat{H}$ is homeomorphic to the Möbius band without boundary. The group $G$ of projective automorphisms of the absolute oval curve $\gamma$ is the fundamental group of transformations for $\widehat{H}, H^{2}$, and the Lobachevskii plane $\Lambda^{2}$.

The research of polygons in the plane $\widehat{H}$ has begun probably in paper [4], where two triangles of different types are represented. Afterwards classical questions on polygons were discussed in relation to the geometry of the plane $\widehat{H}$ in works of many authors; see, for example [1], [2], [3], [7]. In presented work we study regular and equiangular polygons. Relying on results of papers [8], [10], [11], [12], we prove that in the plane $\widehat{H}$ there is no finite regular polygon topologically equivalent to a disk. Each finite equiangular polygon topologically equivalent to a disk is a quadrangle with hyperbolic quasiangles. We study also finite polygons homeomorphic to the Möbius band. We prove that on the plane $\widehat{H}$ the interior angle in a finite equiangular Möbius polygon is a non-convex elliptic pseudoangle. 
Since the geometry of the plane $\widehat{H}$ significantly differs from the geometry of the Lobachevskii plane, especially in its topological component, we adduce in detail all necessary facts in Sec. 2. We pay special attention to the correct introduction of all fundamental notions.

\section{Preliminaries}

\subsection{Angles in the plane $\widehat{H}$}

As in the case of the Lobachevskii plane, there are three types of pencils of lines on $\widehat{H}$. A pencil of lines on $\widehat{H}$ is hyperbolic (elliptic) if its centre is an exterior (interior) point with respect to the absolute. The centre of a parabolic pencil of lines lies on the absolute. Lines of a hyperbolic (elliptic) pencil are said to be intersecting (hyperparallel) on $\widehat{H}$. Lines of a parabolic pencil are said to be parallel. Depending on the types of lines and the type of the pencil containing these lines, a pair of lines determines 15 different types of angles [8, §4.3], [13].

All types of angles of the plane $\widehat{H}$ are presented in Table 1 and in Fig. 1. We denote the hyperbolic, elliptic, and parabolic type of a pencil of lines by symbol $H, E$, and $P$, respectively. For the hyperbolic, elliptic, and parabolic type of a line in the plane $\widehat{H}$ we use the designations $h, e$, and $p$, respectively.

Table 1. The types and the measures of angles on the plane $\widehat{H}$

\begin{tabular}{|c|c|c|c|c|}
\hline \multirow[t]{2}{*}{$\begin{array}{l}\text { Type of an angle } \\
\text { (short designation) }\end{array}$} & \multirow[t]{2}{*}{$\begin{array}{l}\text { Measure } v(\tilde{v}) \text { of } \\
\text { an angle }\end{array}$} & \multirow[t]{2}{*}{$\begin{array}{l}\text { Type of } \\
\text { a pencil }\end{array}$} & \multicolumn{2}{|c|}{$\begin{array}{c}\text { Types } \\
\text { of lines }\end{array}$} \\
\hline & & & $a$ & $b$ \\
\hline Valiana (V) & - & \multirow[t]{2}{*}{$H$} & \multirow[t]{2}{*}{$p$} & \multirow[t]{2}{*}{$p$} \\
\hline Semicovaliana (CV) & - & & & \\
\hline Hyperbolic flag (HF) & - & \multirow[t]{2}{*}{$H$} & \multirow{3}{*}{$p$} & \multirow{3}{*}{$h$} \\
\hline Hyperbolic pseudoflag (HPF) & - & & & \\
\hline Parabolic flag (PF) & - & $P$ & & \\
\hline Elliptic flag (EF) & - & \multirow[t]{2}{*}{$H$} & \multirow[t]{2}{*}{$p$} & \multirow[t]{2}{*}{$e$} \\
\hline Elliptic pseudoflag (EPF) & - & & & \\
\hline Half-plane (HP) & $v \in[0 ; \pi]$ & \multirow{2}{*}{$\begin{array}{l}\frac{E}{H} \\
\end{array}$} & \multirow{5}{*}{$h$} & \multirow{5}{*}{$h$} \\
\hline Hyperbolic angle (HA) & $v \in \mathbb{R}_{+}$or $v_{a} \in \mathbb{R}_{-}$ & & & \\
\hline Hyperbolic pseudoangle (HPA) & $\tilde{v}=i \pi+v, v \in \mathbb{R}_{+}$ & \multirow{3}{*}{$P$} & & \\
\hline Strip (S) & - & & & \\
\hline Pseudostrip (PS) & - & & & \\
\hline Quasiangle (Q) & $\tilde{v}=\varepsilon v+i \pi / 2, v \in \mathbb{R}_{+}, \varepsilon=1 ;-1 ; 0$ & $H$ & $h$ & $e$ \\
\hline Elliptic angle (EA) & $v \in \mathbb{R}_{+}$ & $H$ & $e$ & $e$ \\
\hline Elliptic pseudoangle (EPA) & $\tilde{v}=i \pi-v, v \in \mathbb{R}_{+}$ & & & \\
\hline
\end{tabular}

The angles between non-parallel and non-parabolic lines on the plane $\widehat{H}$ are measurable by means of the absolute. Measurable angles of five types possess vertices in proper domain of the plane $\widehat{H}$. Such angles are elliptic and hyperbolic angles, elliptic and hyperbolic pseudoangles, and quasiangles. The measure of the full angle round a proper point of the plane $\widehat{H}$ equals $2 i \pi$.

An elliptic angle has the real positive measure. A hyperbolic angle has the real negative agreed measure. The measure of an elliptic (hyperbolic) pseudoangle is a complex number $i \pi-v(i \pi+v)$, where $v \in \mathbb{R}_{+}$.

Only non-parabolic lines of different types can be orthogonal on the plane $\widehat{H}$. Two orthogonal lines form two adjacent right quasiangles. The measure of a right quasiangle is equal to $i \frac{\pi}{2}$.

Assume that a hyperbolic line $a$ and an elliptic line $b$ form adjacent quasiangles with the vertex $K$. Let $a^{\prime}$ be an orthogonal line to $a$ and let $K \in a^{\prime}$. The quasiangle between the lines $a$ and $b$ is called hyperbolic (elliptic) if it contains (does not contain) the line $a^{\prime}$. The measure of a hyperbolic (elliptic) quasiangle is a complex number $i \frac{\pi}{2}+\varepsilon v$, where $v \in \mathbb{R}_{+}$and $\varepsilon=1(\varepsilon=-1)$, see [8, §4.5].

\subsection{Polygons on the plane $\widehat{H}$}

Let $\Phi$ be a figure in the plane $H^{2}$. If $\Phi \cap \widehat{H}=\Phi(\Phi \cap \widehat{H} \neq \Phi)$, then the figure $\Phi$ is called a finite (infinite) figure in the plane $\widehat{H}$. 


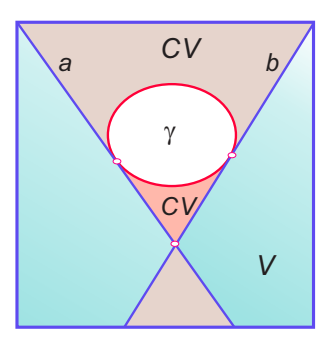

a

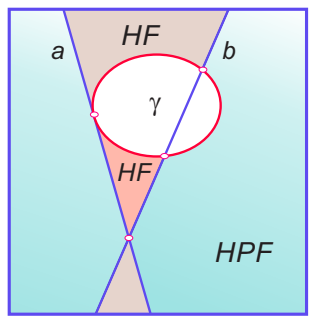

b

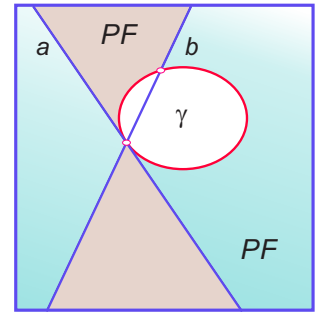

c

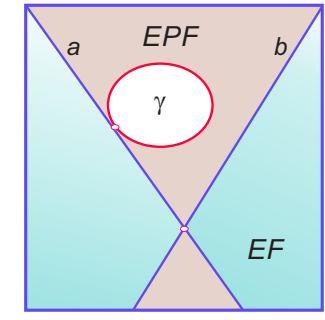

d

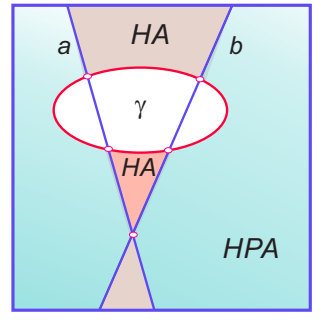

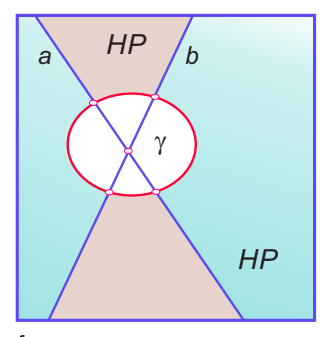

f

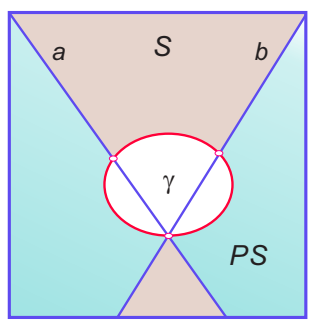

g
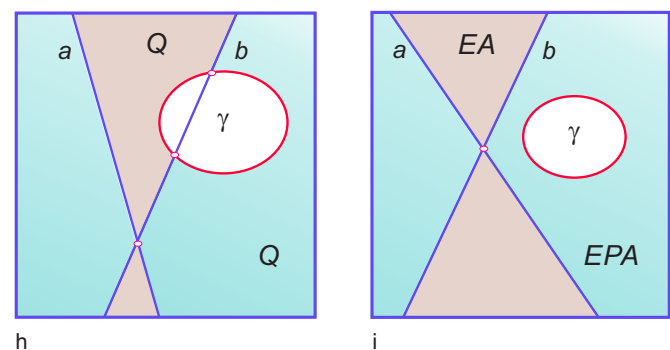

i

Figure 1. The angles of the hyperbolic plane $\widehat{H}$ of positive curvature. The short designations of the types of angles are given in Table 1 .

In some reasonings we suppose that the absolute curve $\gamma$ is added to the extanded hyperbolic plane $H^{2}$. Such gluing the components $\widehat{H}$ and $\Lambda^{2}$ of the plane $H^{2}$ by means of the curve $\gamma$ allow us to simplify introduction of some basic notions. Let $\bar{H}^{2}=H^{2} \cup \gamma$.

On the plane $\bar{H}^{2}$ there are two topological types of simple closed curves. A simple closed curve is called one-sided if its removal from $\bar{H}^{2}$ does not disturb connectivity of this plane. A simple closed curve is called two-sided if it divides the plane $\bar{H}^{2}$ into two connected components.

Let $\partial F$ be a simple two-sided closed broken line on the plane $\bar{H}^{2}$ and let $\partial F \cap \widehat{H} \neq \varnothing$. The broken line $\partial F$ divides the plane $\bar{H}^{2}$ into two connected components. One of these components is topologically equivalent to an open disk, that is, topologically equivalent to the interior domain with respect to an oval curve. We denote it by $o$. The second component is topologically equivalent to the Möbius band without boundary. We denote it by $\mu$. The figure $F_{o}=\partial F \cup o\left(F_{\mu}=\partial F \cup \mu\right)$ is called a generalized polygon (generalized Möbius polygon) of the plane $\widehat{H}$. If the broken line $\partial F$ is finite in $\widehat{H}$, then the generalized polygon $F_{o}$ is also called a polyhedral of the plane $\widehat{H}$.

Vertices and segments of the broken line $\partial F$ are called, respectively, vertices and edges of the polygon with boundary $\partial F$. The line containing a polygon edge is called the side of the polygon. A polygon vertex is called proper or ideal if it belongs to the plane $\widehat{H}$ or the plane $\Lambda^{2}$ respectively. A polygon vertex is called absolute if it lies on the absolute curve $\gamma$. A polygon vertex is true if the polygon edges meeting in it do not lie on one line.

Let us define the notion of the interior angle of a polygon.

Let $F$ be a generalized polygon or a generalized Möbius polygon in the plane $\widehat{H}$ and let all vertices of the polygon $F$ be proper and true. Assume that lines $b$ and $d$ contain the polygon $F$ edges with a common vertex $A$. The lines $b, d$ and the polar line $a$ of the point $A$ with respect to the absolute $\gamma$ divide the plane $\bar{H}^{2}$ into four generalized trihedrals. We call them the basic quarteres of the vertex $A$ and denote by $\eta_{1}, \eta_{2}, \eta_{3}, \eta_{4}$.

Assume that in the plane $\bar{H}^{2}$ an angle $\eta$ between the lines $b$ and $d$ contains the basic quarter $\eta_{q}$ of the vertex $A$, where $q \in\{1,2,3,4\}$. We determine the type of the basic quarter $\eta_{q}$ as the type of $\eta$. If the angle $\eta$ is measurable, we accept its measure as the measure of the basic quarter $\eta_{q}$.

Let $m, n, r$, and $s$ be positive integers and let figures

$$
\eta_{1}^{1}, \ldots, \eta_{1}^{m}, \quad \eta_{2}^{1}, \ldots, \eta_{2}^{n}, \quad \eta_{3}^{1}, \ldots, \eta_{3}^{r}, \quad \eta_{4}^{1}, \ldots, \eta_{4}^{s}
$$

be connected components of intersections $F \cap \eta_{1}, F \cap \eta_{2}, F \cap \eta_{3}$, and $F \cap \eta_{4}$, respectively. The set of figures from (2.1) contains one or three figures possessing a nonempty interior and the point $A$. We denote the quantity of these figures by $Q_{A}$ and we denote the sum of these figures by $F_{A}$. Since all vertices of the polygon $F$ are true, there are two possibilities: $Q_{A}=1$ and $Q_{A}=3$. Let us consider both cases. 
1. If $Q_{A}=1$, then one of the basic quarteres of the vertex $A$ contains the figure $F_{A}$. We call this quarter the interior angle of the polygon $F$ at the vertex $A$.

2. If $Q_{A}=3$, then only one of the basic quarteres of the vertex $A$ does not contain components of the figure $F_{A}$. Let us denote this quarter by $\eta_{4}$. Then each of the basic quarteres $\eta_{1}, \eta_{2}$, and $\eta_{3}$ contains a component from $F_{A}$. We call the sum of the quarteres $\eta_{1}, \eta_{2}$, and $\eta_{3}$ the interior angle of the polygon $F$ at the vertex $A$. Note that we number the basic quarteres so that the quarteres $\eta_{1}$ and $\eta_{3}$ belong to the same type. Under the condition $Q_{A}=3$ the interior angle of the polygon $F$ at the vertex $A$ is non-convex and supplements the quarter $\eta_{4}$ to the plane $\widehat{H}$. Therefore the type name of this angle form from the type name of the quarter $\eta_{4}$ with addition of a term "non-convex". If the angle $\eta$ is measurable, we accept the sum of the measures of the basic quarteres $\eta_{1}, \eta_{2}$, and $\eta_{3}$ as the measure of the interior angle of the polygon $F$ at the vertex $A$.

In Fig. 2, a (b) the interior angle $\eta_{1}\left(\eta_{1} \cup \eta_{2} \cup \eta_{3}\right)$ of the polygon $F$ at the vertex $A$ is shown. In accepted terms, this angle is an elliptic angle (non-convex elliptic angle). For objects in Fig. 2, a (b) we have

$$
\begin{gathered}
F=\eta_{1}^{1} \cup \eta_{2}^{1} \cup \eta_{4}^{1} \cup \eta_{4}^{2} \cup \eta_{4}^{3}, \quad Q_{A}=1, \quad F_{A}=\eta_{1}^{1} \\
\left(F=\eta_{1}^{1} \cup \eta_{1}^{2} \cup \eta_{1}^{3} \cup \eta_{2}^{1} \cup \eta_{3}^{1} \cup \eta_{3}^{2}, \quad Q_{A}=3, \quad F_{A}=\eta_{1}^{1} \cup \eta_{2}^{1} \cup \eta_{3}^{1}\right) .
\end{gathered}
$$

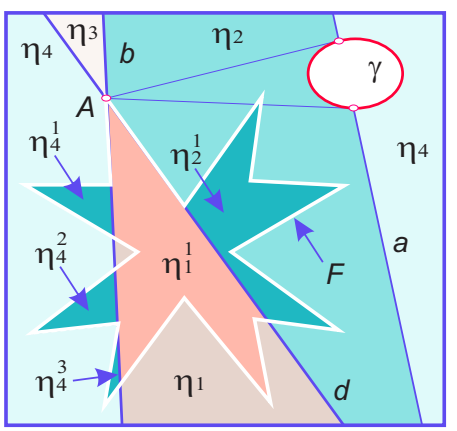

a

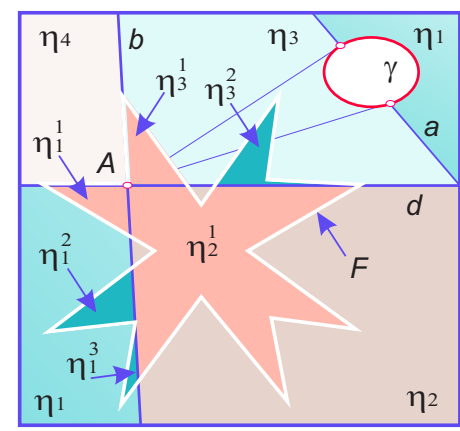

b

Figure 2. (a) The interior angle $\eta_{1}$ of the polygon $F$ at the vertex $A$. (b) The interior angle $\eta_{1} \cup \eta_{2} \cup \eta_{3}$ of the polygon $F$ at the vertex $A$.

A generalized polygon (generalized Möbius polygon) in the plane $\widehat{H}$ is called equiangular if all its interior angles at vertices are congruent. A generalized polygon (generalized Möbius polygon) in the plane $\widehat{H}$ is called regular if all its edges are congruent, and all its interior angles at vertices are congruent.

A generalized polygon (generalized Möbius polygon) whose vertices lie in a cycle is called inscribed polygon in the given cycle.

It is known that there are four types of cycles in the plane $\widehat{H}$. These types are as follows: hypercycles, horocycles, hyperbolic and elliptic cycles; see [5, III, §2], [9, §2.4], [14, §2.4], [15, §2.4]. Only hypercycles are finite in $\widehat{H}$. They can be defined metrically as follows.

Let $S$ be an ideal point on the plane $\widehat{H}$, that is, $S \in \Lambda^{2}$. The set of all points on $\widehat{H}$ such that the hyperbolic distance from it to the point $S$ is a number $r=i \pi \rho / 2-h, h \in \mathbb{R}_{+}$, is called the hypercycle with centre at $S$ and radius $r$. An elliptic line $l$ which is the polar line of the centre of a hypercycle with respect to the absolute curve is called the base of the hypercycle. A hypercycle with base $l$ is the set of all points of the plane $\widehat{H}$ such that the distance from the point to the line $l$ is a real number $h=i \pi \rho / 2-r$, which is called the height of the hypercycle.

The foundation of the areas theory for the plane $\widehat{H}$ is laid in works [10], [11], [12], [16], [17]. The notion of the figure area is entered such that finite figures in the plane $\widehat{H}$ possess the real positive areas. Moreover, for each figure $F$ in the plane $H^{2}$ the following equality holds

$$
S_{\Lambda^{2}}(F)=i S_{\widehat{H}}(F)
$$

where $S_{\Lambda^{2}}(F)$ or $S_{\widehat{H}}(F)$ is the figure $F$ area calculated in geometry of the plane $\Lambda^{2}$ or $\widehat{H}$ respectively; see [17].

We formulate here the theorems about the area of a generalized polygon homeomorphic to a disk and a generalized Möbius polygon in the plane $\widehat{H}$; see [10, Theorem 3.1] and [12, Theorem 1] respectively. 
Theorem 2.1. Let $F_{o}\left(F_{\mu}\right)$ be a generalized polygon (generalized Möbius polygon) without parabolic edges in the plane $\widehat{H}$ of curvature radius $\rho, \rho \in \mathbb{R}_{+}$. Denote the measures of the interior angles of the polygon $F_{o}\left(F_{\mu}\right)$ at vertices $A_{1}, A_{2}$, $\ldots, A_{n}$ by $\widehat{A}_{1}, \widehat{A}_{2}, \ldots, \widehat{A}_{n}$, respectively. Then the area $S_{\widehat{H}}\left(F_{o}\right)\left(S_{\widehat{H}}\left(F_{\mu}\right)\right)$ of the polygon $F_{o}\left(F_{\mu}\right)$ can be expressed by the formula

$$
\begin{gathered}
S_{\widehat{H}}\left(F_{o}\right)=\rho^{2}\left(\sum_{j=1}^{n} \tau_{j} \widehat{A}_{j}-i \pi(n-2)\right) \\
\left(S_{\widehat{H}}\left(F_{\mu}\right)=\rho^{2}\left(\sum_{j=1}^{n} \tau_{j} \widehat{A}_{j}-i \pi n\right)\right),
\end{gathered}
$$

where $\tau_{j}=1$ if a point $A_{j}$ is the proper or absolute vertex of the polygon $F_{o}\left(F_{\mu}\right) ; \tau_{j}=i$ if a point $A_{j}$ is the ideal vertex of the polygon $F_{o}\left(F_{\mu}\right)$.

In this paper we study only finite polygons in which every vertex is true. The areas of such polygons are real numbers.

\section{Property of angles with the common parabolic side}

Let us denote the valiana (covaliana) of an arbitrary point $X$ in the plane $\widehat{H}$ by $W_{X}\left(\widehat{W}_{X}\right)$. At the research of simple $n$-contours in the plane $\widehat{H}$ the following statement is proved; see [8, Lemma 4.3.1].

Lemma 3.1. Let $A$ and $B$ be proper points in the plane $\widehat{H}$ and let $A B, k_{a}$, and $k_{b}$ be distinct parabolic lines such that $A \in k_{a}, B \in k_{b}$, and $K=k_{a} \cap k_{b}$. Then for each point $M$ from the point $K$ valiana the condition $M \in W_{A} \Longleftrightarrow M \in \widehat{W}_{B}$ holds.

In the proof of Theorem 5.2 we use generalization of Lemma 3.1. Let us formulate and prove it in the following lemma.

Lemma 3.2. Assume that in the plane $\bar{H}^{2}$ with the absolute conic $\gamma$ fixed points $A, B, C$ satisfy the following conditions.

(i) $A \notin \gamma$ and $B \notin \gamma$.

(ii) The line $A B$ is parabolic.

(iii) The lines $A C$ and $B C$ of the same type.

Also assume that for a point $M$ in the plane $\bar{H}^{2}$ the point $M_{0}=C M \cap A B$ lies on the segment $A B$. If the point $M$ belongs to the angle $\bar{A}$ between the lines $A B, A C$ and the angle $\bar{B}$ between the lines $A B, B C$, then the angles $\bar{A}$ and $\bar{B}$ of different types.

Proof. Let $A_{1} A_{2}$ (or $A_{1} E$ ) be the polar line of the point $A$ (or $B$, respectively) with respect to the absolute conic $\gamma$, where $A_{1}=A B \cap \gamma, A_{2} \in \gamma, E \in \gamma$; see, for example, Fig. 3, a, where the lines $A C$ and $B C$ are elliptic. Under the conditions $(i),(i i)$ any three of the points $A, A_{1}, A_{2}$, and $E$ do not belong to one line. Consider the canonical frame $R=\left\{A_{1}, A_{2}, A, E\right\}$ of the second type; see [8, §4.1.2]. In the frame $R$ the given points have the following coordinates:

$$
A_{1}(1: 0: 0), A_{2}(0: 1: 0), A(0: 0: 1), E(1: 1: 1), B(2: 0: 1) .
$$

By the lemma conditions, the point $C(M)$ does not lie on the line $A B(0: 1: 0)$. Hence this point can be given in the frame $R$ by coordinates $\left(c_{1}: 1: c_{3}\right)$ (or $\left(m_{1}: 1: m_{3}\right)$ respectively), where $c_{1}, c_{3} \in \mathbb{R}\left(m_{1}, m_{3} \in \mathbb{R}\right)$.

The lines $A A_{2}, A C, B C, B E, A M, B M, C M$ and the point $M_{0}$ have in the frame $R$ the coordinates:

$$
\begin{gathered}
A A_{2}(1: 0: 0), A C\left(-1: c_{1}: 0\right), B C\left(-1: c_{1}-2 c_{3}: 2\right), \\
B E(1: 1:-2), A M\left(-1: m_{1}: 0\right), B M\left(-1: m_{1}-2 m_{3}: 2\right), \\
C M\left(m_{3}-c_{3}: m_{1} c_{3}-m_{3} c_{1}: c_{1}-m_{1}\right), M_{0}\left(m_{1}-c_{1}: 0: m_{3}-c_{3}\right) .
\end{gathered}
$$

Since the point $M_{0}$ lies on the parabolic segment $A B$, for the cross-ratio $\left(A B M_{0} A_{1}\right)$ of four points on the line $A B$ we have the inequality $\left(A B M_{0} A_{1}\right)<0$; see $[8, \S 4.2 .1]$. We write this inequality in the coordinate form:

$$
\left(A B M_{0} A_{1}\right)=\frac{\left|\begin{array}{cc}
0 & 1 \\
m_{1}-c_{1} & m_{3}-c_{3}
\end{array}\right|\left|\begin{array}{cc}
2 & 1 \\
1 & 0
\end{array}\right|}{\left|\begin{array}{cc}
0 & 1 \\
1 & 0
\end{array}\right|\left|\begin{array}{cc}
2 & 1 \\
m_{1}-c_{1} & m_{3}-c_{3}
\end{array}\right|}=\frac{c_{1}-m_{1}}{c_{1}-m_{1}-2\left(c_{3}-m_{3}\right)}<0
$$




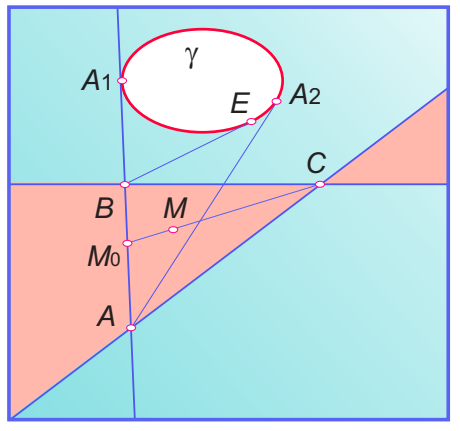

a

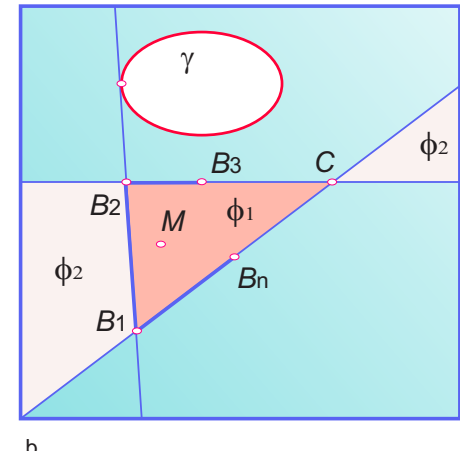

b

Figure 3. (a) The point $M$ belonging to the elliptic pseudoflag between the lines $A B, A C$ and the elliptic flag between the lines $A B, B C$. (b) The parabolic edge $B_{1} B_{2}$ of the polygon $F$. The generalized trihedrals $\varphi_{1}, \varphi_{2}$ between the sides $B_{1} B_{n}$ and $B_{2} B_{3}$.

Let us consider all possibilities depending on type of the lines $A C, B C$.

1. If the lines $A C$ and $B C$ are parabolic, we obtain Lemma 3.1. In this case the lemma statement is proved.

2. Assume that the lines $A C$ and $B C$ are elliptic (or hyperbolic). Then characteristics of these lines, that is, the values of a function $\Phi_{2}=4 X_{1} X_{2}-X_{3}^{2}$ on the real coordinates $\left(-1: c_{1}: 0\right)$ and $\left(-1: c_{1}-2 c_{3}: 2\right)$ of the lines $A C$ and $B C$, respectively, are real numbers of the same sign; see [8, §4.1.2]. Since

$$
\Phi_{2}(A C)=-4 c_{1}, \quad \Phi_{2}(B C)=-4\left(c_{1}-2 c_{3}+1\right),
$$

condition (iii) corresponds to the inequality

$$
c_{1}\left(c_{1}-2 c_{3}+1\right)>0 .
$$

If the lines $A C$ and $B C$ are elliptic (hyperbolic), then each of the angles between the lines $A B, A C$ and $A B, B C$ is an elliptic flag (hyperbolic flag) or, respectively, an elliptic pseudoflag (hyperbolic pseudoflag). By definition, each flag (pseudoflag) does not contain (contains) the parabolic line passing through its vertex; see, for instance, Fig. 1, b, d. Using this condition, we ascertain the types of the angles $\bar{A}$ and $\bar{B}$ containing $M$.

The angle $\bar{A}$ between the lines $A B$ and $A C$ is flag (pseudoflag) if it does not contain (contains) the parabolic line $A A_{2}$. In this case for the cross-ratio $I_{A}=\left((A B)(A C)(A M)\left(A A_{2}\right)\right)$ of the quadruple of lines from the pensil with centre at $A$ we have the inequality $I_{A}<0\left(I_{A}>0\right)$. Similarly, the angle $\bar{B}$ between the lines $A B$ and $B C$ is flag (pseudoflag) if it does not contain (contains) the parabolic line $B E$. In this case for the cross-ratio $I_{B}=((A B)(B C)(B M)(B E))$ of the quadruple of lines from the pensil with centre at $B$ we have the inequality $I_{B}<0\left(I_{B}>0\right)$. We express the values of $I_{A}$ and $I_{B}$ through the parameters $c_{1}, c_{3}, m_{1}, m_{3}$ :

$$
\begin{gathered}
I_{A}=\frac{\left|\begin{array}{cc}
0 & 1 \\
-1 & m_{1}
\end{array}\right| \begin{array}{cc}
-1 & c_{1} \\
1 & 0
\end{array} \mid}{\left|\begin{array}{cc}
0 & 1 \\
1 & 0
\end{array}\right|\left|\begin{array}{cc}
-1 & c_{1} \\
-1 & m_{1}
\end{array}\right|}=\frac{c_{1}}{c_{1}-m_{1}}, \\
I_{B}=\frac{\left|\begin{array}{ccc}
0 & 1 \\
1 & 2 m_{3}-m_{1}
\end{array}\right|\left|\begin{array}{cc}
1 & 2 c_{3}-c_{1} \\
1 & 1
\end{array}\right|}{\left|\begin{array}{cc}
0 & 1 \\
1 & 1
\end{array}\right|\left|\begin{array}{cc}
1 & 2 c_{3}-c_{1} \\
1 & 2 m_{3}-m_{1}
\end{array}\right|}=\frac{c_{1}-2 c_{3}+1}{c_{1}-m_{1}-2\left(c_{3}-m_{3}\right)} .
\end{gathered}
$$

Due to conditions (3.1), (3.2) the inequality $I_{A} I_{B}<0$ holds. This means that the angles $\bar{A}$ and $\bar{B}$ of different types.

The lemma is proved.

\section{General property of finite equiangular polygons of the plane $\widehat{H}$}

Lemma 4.1. A finite equiangular polygon of the plane $\widehat{H}$ has no parabolic edges. 
Proof. Assume that in the plane $\widehat{H}$ there is a finite equiangular polygon $F$. Let us show that the polygon $F$ has no parabolic edges.

Let points $B_{1}, \ldots, B_{n}$ be the vertices of the polygon $F$. Assume antithesis. Agree that in the polygon $F$ an edge, for example, $B_{1} B_{2}$, is a parabolic segment. Denote the intersection point of the sides $B_{1} B_{n}$ and $B_{2} B_{3}$ by $C$; see, for instance, Fig. 3, b, where the case with the elliptic sides $B_{1} B_{n}$ and $B_{2} B_{3}$ of the polygon $F$ is shown. Since the polygon $F$ is equiangular, the lines $B_{1} B_{n}$ and $B_{2} B_{3}$ of the same type. These lines divide the plane $\bar{H}^{2}$ into two angles. One of them contains the segment $B_{1} B_{2}$ wich divides this angle into two generalized trihedrals. Denote them by $\varphi_{1}$ and $\varphi_{2}$. If the edges $B_{1} B_{n}$ and $B_{2} B_{3}$ belong to the boundaries of the different trihedrals $\varphi_{1}$ and $\varphi_{2}$, then only one of the interior angles of the polygon $F$ at the vertices $B_{1}$ and $B_{2}$ is convex. It contradicts assumption about equality of all angles in the polygon $F$. Consequently, the points $B_{n}$ and $B_{3}$ belong to the boundary of one of the trihedrals $\varphi_{1}, \varphi_{2}$. Denote this trihedral by $\varphi_{1}$. If the interior angle of the polygon $F$ at every vertex is convex (non-convex), then in the interior of the trihedral $\varphi_{1}\left(\varphi_{2}\right)$ there is a point $M$ wich belongs to the interior angles of $F$ at the vertices $B_{1}$ and $B_{2}$. Since a point $M_{0}=C M \cap B_{1} B_{2}$ belongs to the parabolic segment $B_{1} B_{2}$, the points $B_{1}, B_{2}, C$, and $M$ satisfy the Lemma 3.2 conditions. By Lemma 3.2, the interior angles of the polygon $F$ at the vertices $B_{1}$ and $B_{2}$ are not congruent. We reached a contradiction. Consequently, our assumption is incorrect, and the polygon $F$ has no parabolic edges.

The lemma is proved.

\section{Equiangular and regular polygons homeomorphic to a disk}

Theorem 5.1. If in the plane $\widehat{H}$ there is a finite equiangular polygon homeomorphic to a disk, then it is a quadrangle with hyperbolic quasiangles.

Proof. Assume that in the plane $\widehat{H}$ there is a finite equiangular $n$-gon $F_{o}$ homeomorphic to a disk. By Lemma 4.1, the polygon $F_{o}$ has no parabolic edges. Consequently, the interior angles of $F_{o}$ at all vertices are measurable.

Denote the measure of the interior angle of $F_{o}$ at every vertex by $\beta$. Since the polygon $F_{o}$ is finite, all its vertices lie in the plane $\widehat{H}$. Via formula (2.2) from Theorem 2.1 we find

$$
S_{\widehat{H}}\left(F_{o}\right)=\rho^{2}(n \beta-i \pi(n-2)) \text {. }
$$

In the plane $\widehat{H}$ a measurable angle with finite vertex belongs to one of five types; see Table 1 . Let us consider all possibilities depending on the types of interior angles in the polygon $F_{o}$.

1. Assume that the interior angle of the polygon $F_{o}$ at every vertex is convex.

(a) If each interior angle of the polygon $F_{o}$ is an elliptic or hyperbolic angle, then $\beta \in \mathbb{R}$. In these cases the area $S_{\widehat{H}}\left(F_{o}\right)$ calculated via formula (5.1) is not real number. Therefore the polygon $F_{o}$ is infinite and does not conform to the theorem requirement.

(b) If each interior angle of the polygon $F_{o}$ is an elliptic or hyperbolic pseudoangle, then $\beta=i \pi-\beta_{0}$, where $\beta_{0} \in \mathbb{R}_{+}$or $\beta_{0} \in \mathbb{R}_{-}$, respectively. In these cases from (5.1) we obtain: $S_{\widehat{H}}\left(F_{o}\right) \in \mathbb{C}$ and $\operatorname{Im}\left(S_{\widehat{H}}\left(F_{o}\right)\right)=2 \pi \rho^{2} \neq 0$. Hence the polygon $F_{o}$ is infinite and does not satisfy the theorem condition.

(c) Let us agree that each interior angle of the polygon $F_{o}$ is a quasiangle. Then $\beta=i \frac{\pi}{2}+\varepsilon \beta_{0}$, where $\beta_{0} \in \mathbb{R}_{+}, \varepsilon=0 ; 1 ;-1$. Formula (5.1) yields

$$
S_{\widehat{H}}\left(F_{o}\right)=\rho^{2}\left(i \pi \frac{4-n}{2}+n \varepsilon \beta_{0}\right) .
$$

From expression (5.2) we find the values of $n, \varepsilon$ for the finite polygon $F_{o}: n=4, \varepsilon=1$. Thus an equiangular polygon with quasiangles is finite if and only if it is a quadrangle with hyperbolic quasiangles.

2. Assume that the interior angle of the polygon $F_{o}$ at every vertex is non-convex. Then it can be an angle of only one of five types. Let us consider all alternatives.

(a) If each interior angle of the polygon $F_{o}$ is a non-convex elliptic or hyperbolic angle, then $\beta=2 i \pi-\beta_{0}$, where $\beta_{0} \in \mathbb{R}_{+}$or $\beta_{0} \in \mathbb{R}_{-}$respectively. By formula (5.1) we obtain

$$
S_{\widehat{H}}\left(F_{o}\right)=\rho^{2}\left(i \pi(n+2)-n \beta_{0}\right) .
$$


Under admissible values $n$ the number $S_{\widehat{H}}\left(F_{o}\right)$ from (5.3) is not real. Therefore the polygon $F_{o}$ is infinite. It is not provided by the theorem.

(b) If each interior angle of the polygon $F_{o}$ is a non-convex elliptic or hyperbolic pseudoangle, then $\beta=i \pi+\beta_{0}$, where $\beta_{0} \in \mathbb{R}_{+}$or $\beta_{0} \in \mathbb{R}_{-}$respectively. In these cases formula (5.1) implies

$$
S_{\widehat{H}}\left(F_{o}\right)=\rho^{2}\left(2 i \pi+n \beta_{0}\right) .
$$

Since $\operatorname{Im}\left(S_{\widehat{H}}\left(F_{o}\right)\right)=2 \pi \rho^{2} \neq 0$, the polygon $F_{o}$ is infinite. It contradicts the theorem condition.

(c) If each interior angle of the polygon $F_{o}$ is a non-convex quasiangle, then $\beta=i \frac{3 \pi}{2}+\varepsilon \beta_{0}$, where $\beta_{0} \in \mathbb{R}_{+}, \varepsilon=0 ; 1 ;-1$. Formula (5.1) implies

$$
S_{\widehat{H}}\left(F_{o}\right)=\rho^{2}\left(i \pi \frac{4+n}{2}+n \varepsilon \beta_{0}\right) .
$$

Under admissible values $n$ we have $\operatorname{Im}\left(S_{\widehat{H}}\left(F_{o}\right)\right) \neq 0$. Hence the polygon $F_{o}$ is infinite. This case violates the theorem requirement and completes consideration of all possibilities.

Note that the only case 1 (c) satisfies the theorem conditions. So in the plane $\widehat{H}$ only a quadrangle with hyperbolic quasiangles can be a finite equiangular polygon homeomorphic to a disk.

The theorem is proved.

The existence of a finite equiangular quadrangle can be proved via its explicit construction. For this purpose we can use a simple 4-contour or a Lambert quadrilateral in the plane $\widehat{H}$; see [18] or [6] respectively.

In Fig. 4, a, the finite equiangular quadrangle $A B C D$ is shown.

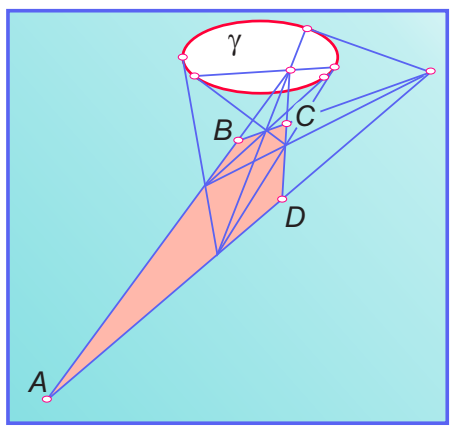

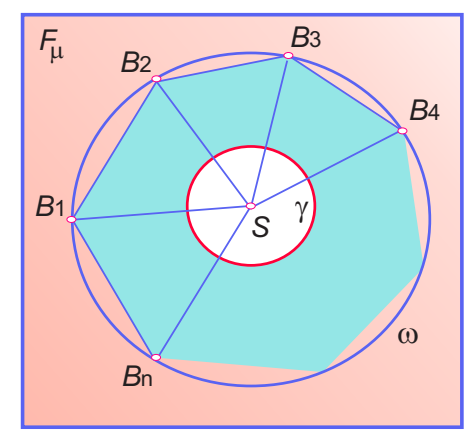

b

Figure 4. (a) The finite equiangular quadrangle $A B C D$. (b) The finite regular Möbius polygon $F_{\mu}=B_{1} B_{2} \ldots B_{n}$ inscribed in the hypercycle $\omega$.

Using a coordinate method, it is possible to prove the following assertions.

1. In the plane $\widehat{H}$ the opposite edges of a finite equiangular quadrangle are congruent.

2. In the plane $\widehat{H}$ the midlines of a finite equiangular quadrangle are mutually orthogonal and have common midpoint.

Theorem 5.1 allows us to prove the following fact.

Theorem 5.2. In the plane $\widehat{H}$ there is no finite regular poligon homeomorphic to a disk.

Proof. Assume that in the plane $\widehat{H}$ there is a finite regular polygon $F$ homeomorphic to a disk. By definition, all interior angles of the polygon $F$ are congruent. Hence for the polygon $F$ we have Theorem 5.1. By this theorem, the polygon $F$ is a finite quadrangle with hyperbolic quasiangles. The sides of a quasiangle belong to different types of lines. Therefore adjacent edges of the polygon $F$ are not congruent. Thus we obtain the violation to the theorem condition. Consequently, our assumption is incorrect.

The theorem is proved. 


\section{Equiangular and regular Möbius polygons}

Theorem 6.1. In the plane $\widehat{H}$ the interior angle at the vertex of a finite equiangular Möbius polygon is a non-convex elliptic pseudoangle.

Proof. Let $F_{\mu}$ be a finite equiangular Möbius $n$-gon in the plane $\widehat{H}$. By Lemma 4.1 , the polygon $F_{\mu}$ has no parabolic edges. Therefore $F_{\mu}$ satisfies the Theorem 2.1 conditions. Formula (2.3) from Theorem 2.1 yields the expression

$$
S_{\widehat{H}}\left(F_{\mu}\right)=\rho^{2} n(\beta-i \pi),
$$

where $\beta$ is the measure of each interior angle in the polygon $F_{\mu}$. The values of $S_{\widehat{H}}\left(F_{\mu}\right)$ are real and positive if and only if $\beta=i \pi+v, v \in \mathbb{R}_{+}$.

Assume that the polygons $F_{\mu}, F_{o}$ have a common boundary. Then the generalized equiangular polygon $F_{o}$ is topologically equivalent to a disk. All interior angles at vertices in such polygon are convex. For the measure $\beta_{o}$ of each interior angle of $F_{o}$ we obtain: $\beta_{o}=2 i \pi-\beta=i \pi-v$. Choosing the values of the measures of convex angles from Table 1, we find the only possibility: the interior angle of the polygon $F_{o}$ is an elliptic pseudoangle. Therefore the interior angle of the polygon $F_{\mu}$ is a non-convex elliptic pseudoangle.

The theorem is proved.

Before proving the existence of a finite regular Möbius polygon in the plane $\widehat{H}$, we prove some of its properties.

Theorem 6.2. In the plane $\widehat{H}$ of curvature radius $\rho, \rho \in \mathbb{R}_{+}$, vertices of a finite regular Möbius n-gon with the edge length a lie in a hypercycle with the height $h$, where

$$
h=\rho \ln \frac{\sin \frac{a}{2 \rho}+\sqrt{\sin ^{2} \frac{a}{2 \rho}-\sin ^{2} \frac{\pi}{n}}}{\sin \frac{\pi}{n}} .
$$

The length a of each edge in a finite regular Möbius n-gon satisfies the inequality

$$
a>2 \pi \rho / n \text {. }
$$

Proof. Assume that in the plane $\widehat{H}$ there is a finite regular Möbius $n$-gon $F_{\mu}$. Denote its vertices by $B_{1}, B_{2}$, $\ldots, B_{n}$. Owing to Theorem 6.1 all edges of the polygon $F_{\mu}$ are elliptic segments. For each elliptic segment of the plane $\widehat{H}$ there exists the unique midperpendicular. Let $p_{1}$ and $p_{2}$ be the midperpendiculars of the edges $B_{1} B_{2}$ and $B_{2} B_{3}$ respectively, and let $S=p_{1} \cap p_{2}$. Then the vertices $B_{1}, B_{2}$, and $B_{3}$ are at the same distance from the point $S$. Therefore these vertices lie in a cycle with centre at $S$. Denote this cycle by $\omega$. If $n>3$ then we continue reasonings. Since $\left|S B_{1}\right|=\left|S B_{2}\right|=\left|S B_{3}\right|$ and $\left|B_{1} B_{2}\right|=\left|B_{2} B_{3}\right|$, the generalized trihedrals $B_{1} B_{2} S$, $B_{2} B_{3} S$ are congruent. Notice that a priori depending on location of the point $S$ we can have one of the following conditions:

1) $\left|S B_{1}\right| \in \mathbb{R}_{+}$if $S \in \widehat{H}$;

2) $\left|S B_{1}\right|=\infty$ if $S \in \gamma$;

3) $\left|S B_{1}\right|=i \pi / 2-v$, where $v \in \mathbb{R}_{+}$, if $S \in \Lambda^{2}$.

As the interior angles at the vertices $B_{2}$ and $B_{3}$ of the polygon $F_{\mu}$ are congruent and $\left|B_{2} B_{3}\right|=\left|B_{3} B_{4}\right|$, the generalized trihedrals $B_{2} B_{3} S, B_{3} B_{4} S$ are also congruent. Hence $\left|S B_{4}\right|=\left|S B_{1}\right|$ and $B_{4} \in \omega$. Continuing sequentially similar resonings, we prove that every vertex of the polygon $F_{\mu}$ belongs to the cycle $\omega$. Further we ascertain the type of $\omega$.

The midperpendicular of each polygon $F_{\mu}$ edge is a hyperbolic line. Therefore the lines $S B_{1}, S B_{2}, \ldots, S B_{n}$, that is, axes of $\omega$, are hyperbolic too. It means that the cycle $\omega$ can be a horocycle, a hyperbolic cycle, or a hypercycle of the plane $\widehat{H}$. Let us choose the round $\xi$ of the cycle $\omega$ according to the cyclic sequence of vertices $B_{1}, B_{2}, \ldots, B_{n}$ of the polygon $F_{\mu}$. The round $\xi$ generates orientation of the plane $\widehat{H}$. In $[9, \S \S 3.5 .1,3.5 .2,3.6 .4]$ it is shown that in the oriented plane $\widehat{H}$ angles with hyperbolic sides are oriented.

Let $f$ be a rotation through an oriented angle $B_{1} S B_{2}$ around the point $S$. Clearly, $f(\omega)=\omega$. According to the previous arguments, the trihedrals $B_{1} B_{2} S, B_{2} B_{3} S, \ldots, B_{n} B_{1} S$ are congruent. Hence oriented angles $B_{1} S B_{2}, B_{2} S B_{3}, \ldots, B_{n} S B_{1}$ are also congruent. Owing to this for any numbers $p$ and $q$, where $p=1,2, \ldots, n$ and $q=1,2, \ldots, n$, there exists a number $m$ satisfying the condition

$$
f^{m}\left(B_{p}\right)=B_{q} .
$$


Since the rotation $f$ keeps orientation of the plane $\widehat{H}$, the condition (6.3) implies that the cycle $\omega$ does not possess points from the absolute. Consequently, the cycle $\omega$ is a hypercycle of the plane $\widehat{H}$; see Fig. 4 , b.

On the plane $\widehat{H}$ a hypercycle of height $h$ posseses the following property; see [19, Theorem 1]. The length $a$ of its interior elliptic chord corresponding to the central angle of the measure $\alpha$ is determined by the equality

$$
\cos \frac{a}{\rho}=1-2 \sin ^{2} \frac{\alpha}{2} \cosh ^{2} \frac{h}{\rho} .
$$

In the case of the regular $n$-gon $F_{\mu}$, inscribed in the hypercycle $\omega$ of height $h$, we have $\alpha=2 \pi / n$. Therefore via the formula (6.4) we find

$$
\begin{gathered}
\cos \frac{a}{\rho}=1-2 \sin ^{2} \frac{\pi}{n} \cosh ^{2} \frac{h}{\rho} \\
\cos ^{2} \frac{a}{2 \rho}-\sin ^{2} \frac{a}{2 \rho}=\cos ^{2} \frac{a}{2 \rho}+\sin ^{2} \frac{a}{2 \rho}-2 \sin ^{2} \frac{\pi}{n} \cosh ^{2} \frac{h}{\rho} \\
\sin ^{2} \frac{a}{2 \rho}=\sin ^{2} \frac{\pi}{n} \cosh ^{2} \frac{h}{\rho} .
\end{gathered}
$$

Since $a \in(0, \pi \rho), h \in \mathbb{R}_{+}$, and $n \geq 3$, formula (6.5) implies

$$
\cosh \frac{h}{\rho}=\frac{\sin \frac{a}{2 \rho}}{\sin \frac{\pi}{n}}
$$

Owing to equality (6.6) we obtain

$$
e^{\frac{h}{\rho}}=\frac{\sin \frac{a}{2 \rho}+\sqrt{\sin ^{2} \frac{a}{2 \rho}-\sin ^{2} \frac{\pi}{n}}}{\sin \frac{\pi}{n}} \text { or } e^{\frac{h}{\rho}}=\frac{\sin \frac{a}{2 \rho}-\sqrt{\sin ^{2} \frac{a}{2 \rho}-\sin ^{2} \frac{\pi}{n}}}{\sin \frac{\pi}{n}} .
$$

For all admissible values of $a$ and $n$ the following inequality holds

$$
\frac{\sin \frac{a}{2 \rho}-\sqrt{\sin ^{2} \frac{a}{2 \rho}-\sin ^{2} \frac{\pi}{n}}}{\sin \frac{\pi}{n}}<1 .
$$

Hence the second equality from (6.7) contradicts the condition $h \in \mathbb{R}_{+}$. According to the first equality from (6.7), we have (6.1). This completes the proof of the first theorem statement.

Since $\cosh \frac{h}{\rho}>1$, from the equality (6.6) we obtain

$$
\sin \frac{a}{2 \rho}>\sin \frac{\pi}{n}
$$

Consequently, the inequality (6.2) holds. So, the second statement of the theorem is proved.

The theorem is proved.

Using Theorem 6.2, it is possible to prove the existence of a finite regular Möbius polygon of the plane $\widehat{H}$ via an explicit construction.

A regular $n$-contour considered in [20], [21, §4] (see also [9, §5.2]) at construction of simple partitions of the plane $\widehat{H}$ gives us interesting examples of finite regular Möbius polygons. It is known that on the plane $\widehat{H}$ a regular $n$-contour defines a family of concentric hypercycles. For $n$ odd this family consists of $(n-1) / 2$ nondegenerate hypercycles, and for $n$ even it consists of $(n-2) / 2$ nondegenerate hypercycles. All vertices of the same order on a regular $n$-contour are located at the same hypercycle.

Assume that points $A_{1}^{m}, A_{2}^{m}, \ldots, A_{n}^{m}$ are vertices of order $m, m>1$, on a regular $n$-contour with centre at a point $S$. Let us consider a rotation of the plane $\widehat{H}$ through the angle $2 \pi / n$ about the point $S$. Under this rotation every point $A_{j}^{m}$ goes to $A_{j+1}^{m}$, where $A_{n t+j}^{m}=A_{j}^{m}, j, t \in \mathbb{N}$. Consequently, a closed broken line $A_{1}^{m} A_{2}^{m} \ldots A_{n}^{m}$ defines two regular polygons. Since $m>1$, each line $A_{j}^{m} A_{j+1}^{m}$ is elliptic. Hence the regular Möbius polygon $F$ with the vertices $A_{1}^{m}, A_{2}^{m}, \ldots, A_{n}^{m}$ is finite.

Thus a regular $n$-contour of the plane $\widehat{H}$ generates a family of regular Möbius polygons. For $n$ odd this family consists of $((n-1) / 2-1) n$-gons, and for $n$ even it consists of $((n-2) / 2-1) n$-gons. 
Note that regular $n$-contours in the plane $\widehat{H}$ at odd and even $n$ possess different combinatory structures; see, for instance, [21, Sec. 4.5]. Distinctions under odd and even $n$ are found also in a combinatory structure of the finite regular Möbius polygons of the plane $\widehat{H}$. For example, if $n$ is odd, a finite regular Möbius $n$-gon consists of $n$ congruent trihedrals which fit together on a common edge without gaps and overlappings. For $n$ even the cells of patitions in a finite regular Möbius $n$-gon are $n / 2$ congruent equiangular quadrangles.

In Fig. 5 a finite regular Möbius 9-gon $F$ with vertices $1,2, \ldots, 9$ is shown. The polygon $F$ is inscribed in a hypercycle $\omega$. An elliptic line $l$ is the base of $\omega$. The trihedrals $Q_{1}=162, Q_{2}=627, Q_{3}=273, Q_{4}=738, Q_{5}=384$, $Q_{6}=849, Q_{7}=495, Q_{8}=951, Q_{9}=516$ form the polygon $F$.

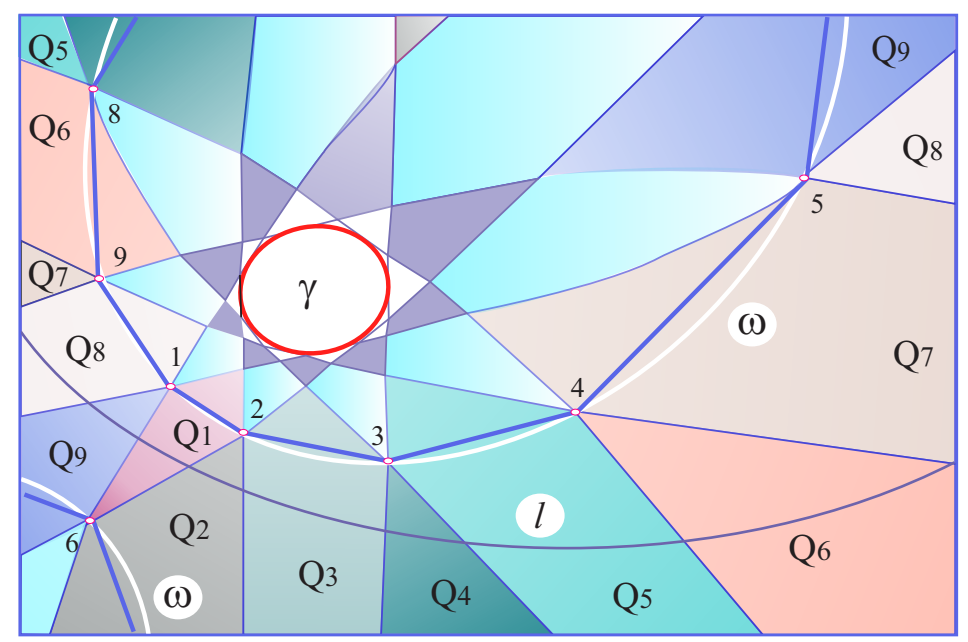

Figure 5. The finite regular Möbius 9-gon $F=12 \ldots 9$ generated by a regular 9-contour. The vertices $1,2, \ldots, 9$ of $F$ lie in a hypercycle $\omega$ with the base $l$. The trihedrals $Q_{1}=162, Q_{2}=627, Q_{3}=273, Q_{4}=738, Q_{5}=384, Q_{6}=849, Q_{7}=495, Q_{8}=951, Q_{9}=516$ form the polygon $F$.

\section{References}

[1] Asmus, Im., Duality between hyperbolic and de Sitter geometry. J. Geom. 96 (2009), no. 1-2, 11-40.

[2] Böhm, J. and Hof, H. C. I., Flächeninhalt verallgemeinerter hyperbolischer Dreiecke. Geom. Dedicata 42 (1992), no. 2, $223-233$.

[3] Cho, Y.-H., Trigonometry in extended hyperbolic space and extended de Sitter space. Bull. Korean Math. Soc. 46 (2009), no. 6, $1099-1133$.

[4] Coxeter, H. S. M., A Geometrical Background for De Sitter's World. Amer. Math. Mon. 50 (1943), no. 4, 217-228.

[5] Klein, F., Vorlesungen Über Nicht-Euclidische Geometrie. Verlag Von Julius Springer, Berlin, 1928.

[6] Klychkova, V. N., Trirectangle of the hyperbolic plane of positive curvature. Coll. of articles of the Int. sci. and pract. conf. "Modern view on the future of science", Tomsk, May 25, 2016. Aeterna. (2016), 14-17. (In Russian)

[7] Milnor, J. W., Hyperbolic geometry: The first 150 years. Amer. Math. Soc. (N.S.). 6 (1982), no. 1, 9-24.

[8] Romakina, L. N., Geometry of the hyperbolic plane of positive curvature. P. 1: Trigonometry. Publishing House of the Saratov University, Saratov, 2013. (In Russian)

[9] Romakina, L. N., Geometry of the hyperbolic plane of positive curvature. P. 2: Transformations and Simple Partitions. Publishing House of the Saratov University, Saratov, 2013. (In Russian)

[10] Romakina, L. N., The area of a generalized polygon without parabolic edges of a hyperbolic plane of positive curvature. Asian J. Math. Comp. Research. 10 (2016), no. 4, 293-310.

[11] Romakina, L. N., On the area of a trihedral on a hyperbolic plane of positive curvature. Mat. Tr. 17 (2014), no. 2, 184-206. (In Russian). Translated in Sib. Adv. Math. 25 (2015), no. 2, 138-153.

[12] Romakina, L. N., The areas of the regular polygons, inscribed in a hypercycle of a hyperbolic plane of positive curvature. Int. Sci. J. «Innovative science». 6 (2016), 20-22. (In Russian)

[13] Romakina, L. N., Analogs of a formula of Lobachevsky for angle of parallelism on the hyperbolic plane of positive curvature. Sib. Elektron. Mat. Izv. 10 (2013), 393-407. (In Russian)

[14] Romakina, L. N., Oval lines of a hyperbolic plane of positive curvature. Izv. Saratov Univ. (N.S.), Ser. Math. Mech. Inform. 12 (2012), no. 3, 37-44. (In Russian)

[15] Romakina, L. N., Cycles of the hyperbolic plane of positive curvature. Zap. Nauchn. Sem. POMI. 415 (2013), 137-162. (In Russian). Translated in J. Math. Sci. 212 (2016), no. 5, 605-621.

[16] Romakina, L. N., The theorem of the area of a rectangular trihedral of the hyperbolic plane of positive curvature. Dal'nevost. Mat. Zh. 13 (2013), no. 1, 127-147. (In Russian)

[17] Romakina, L. N., To the theory of the areas of a hyperbolic plane of positive curvature. Publications de l'Institut Mathematique. 99 (2016), no. 113, 139-154. (In Russian).

[18] Romakina, L. N., Finite closed 3(4)-loops of extended hyperbolic plane. Izv. Saratov Univ. (N.S.), Ser. Math. Mech. Inform. 10 (2010), no. 3, 14-26. (In Russian) 
[19] Romakina, L. N., The chord length of a hypercycle in a hyperbolic plane of positive curvature. Sibirsk. Mat. Zh. 54 (2013), no. 5, $1115-1127$. (In Russian). Translated in Sib. Math. J. 54 (2013), no. 5, 894-904.

[20] Romakina, L. N., Partitions of a hyperbolic plane of positive curvature generated by a regular n-contour. Relativity theory, gravity and geometry, Proc. of the Petrov 2010 Anniversary Symposium on General Relativity and Gravitation (Kazan' 2010), Kazan' State University, Kazan' (2010). 227-232. (In Russian)

[21] Romakina, L. N., Simple partitions of a hyperbolic plane of positive curvature. Mat. Sb. 203 (2012), no. 9, 83-116. (In Russian). Translated in Sbornik: Mathematics. 203 (2012), no. 9, 1310-1341.

[22] Rosenfel'd, B. A., Zamakhovskii, M. P., Geometry of Lie groups. Symmetric, parabolic and periodic spaces. Moscow centre for Countinuous Mathematical Education, Moscow, 2003. (In Russian)

[23] Rosenfel'd, B. A., Noneuclidean spaces. Nauka, Moscow, 1969. (In Russian)

[24] De Sitter, W., On the Relativity of Inertia. Remarks Concerning Einstein's Latest Hypothesis. Proc. Royal Acad. Amsterdam. 19 (1917), no. 2, 1217-1225.

\section{Affiliations}

LYUDMILA N. ROMAKINA

ADDRESS: Saratov State University, Dept. of Geometry, Russia.

E-MAIL: romakinaln@mail.ru

ORCID ID : orcid.org/0000-0002-3695-2076 